\title{
Understanding and predicting fracture risk in acromegaly
}

\author{
Natasha M. Appelman-Dijkstra ${ }^{1,2} \cdot$ Nienke R. Biermasz $^{1}$
}

Received: 30 December 2016 / Accepted: 17 January 2017 / Published online: 2 February 2017

(C) Springer Science+Business Media New York 2017

Acromegaly is a rare endocrine disease that has been shown to be associated with an increased risk of vertebral fractures even in the presence of a relatively normal bone mineral density (BMD). These fractures do not only occur during the period of active acromegaly, but it has been shown that after remission of acromegaly patients continue to have an increased risk for new and progression of vertebral fractures, see a recent review [1].

At present the pathophysiological factors determining bone fragility in patients with acromegaly, are not wellelucidated, due to the complex combinations of endocrine conditions. These conditions include the variable severity and duration of growth hormone $(\mathrm{GH})$ excess and variable concomitant endocrine dysfunction, such as hypogonadism or hypocortisolism, and the onset of disease in different stages of skeletal development, i.e., early adulthood or at older age. Well-designed prospective research is limited by the rarity of the disease and the limitations of standard imaging techniques, i.e., dual-energy X-ray absorptiometry, to reflect bone fragility and to predict fracture risk in this patient group.

In general, patients with active acromegaly demonstrate high bone turnover associated with an increase in cortical BMD in the presence of stable trabecular bone mass. Iliac crest biopsies of acromegaly patients indeed show increased

Nienke R. Biermasz

n.r.biermasz@lumc.nl

1 Leiden University Medical Center, Center for Endocrine Tumors Leiden, Leiden, The Netherlands

2 Department of Internal Medicine: division Endocrinology, Center for Bone Quality Leiden, Leiden, The Netherlands bone remodeling compared to healthy controls [2]. In these biopsies due to the high bone turnover, trabecular connections are lost resulting in altered microarchitecture. These microarchitectural changes persist after successful induction of remission and restoration of bone remodeling rates. In a study with HRpQCT, structural measurements confirmed this altered bone microarchitecture in patients with active, as well as controlled acromegaly, acknowledging hypogonadism as a relevant determinant [3]. These structural changes included deteriorated trabecular microarchitectural parameters in the distal radius and distal tibia, and increased cortical volumetric BMD in the distal tibia in patients with active disease. In this study, the relation of these parameters with fracture or fracture risk was not assessed. Areal BMD is often reported to be normal in these patients.

Overall, current imaging methods do not provide an explanation for the reported increased vertebral fracture risk in acromegaly patients.

In this issue, a new study by Maffezzoni and collegues [4] report on bone microarchitectural changes in acromegaly patients in remission. They evaluate the ability of highresolution cone-beam computed tomography in acromegaly to provide information on skeletal abnormalities and were the first to associate this with vertebral fractures in 40 patients and 21 healthy non-matched controls. They found that acromegaly patients with vertebral fractures (15/40) had lower bone volume/trabecular volume ratio, greater mean trabecular separation and higher cortical porosity compared to non-fracture acromegaly patients. Furthermore they report that all patients with acromegaly showed lower bone volume/trabecular volume ratio, mean trabecular thickness and greater mean trabecular separation than control subjects. These findings are interesting since they provide more answers to the long asked question how to identify 
acromegaly patients at risk for vertebral fractures. Recent cross-sectional and longitudinal studies have shown altered trabecular bone scores (TBS) in patients with acromegaly as well, where it was even shown that during follow up after treatment initiation a decrease in TBS occured. Vallassi et al. [5] reported that both cortical and trabecular volumetric BMD (vBMD) are reduced at the proximal femur in acromegaly patients, regardless of gender, gonadal status, and disease activity. Volumetric BMD is used to measure the bone mineral density of only the spongy interior bone separately from the cortical bone and is not affected by changes in size of bones. Disease duration was negatively associated with trabecular vBMD at the total hip.

After remission of acromegaly, vertebral fractures are still highly prevalent and do progress in $20 \%$ of patients suggesting permanent alterations of bone structure. Although the vertebral fractures are well explained due to the loss of trabecula's during the active phase, prospective studies show that patients with incident vertebral fractures do have a significant reduction in BMD at the femoral neck during follow-up, implying increased cortical bone loss at follow up in fracture patients, a difference also noticed in this study by Maffezzoni et al.

However, one has to bear in mind that despite all the suggestions of alterations in bone microarchitecture there are hardly published data on the assessment of material properties of bone in patients with acromegaly. Currently there are no treating guidelines addressing the skeletal complications of acromegaly nor is there treatment consensus on how to prevent vertebral fractures in this rare disease. Based on the current literature clearly showing microarchitectural changes in the different skeletal sites in acromegaly patients, the focus of future research should divert toward patient related outcomes like fracture incidence or morbidity and treatment options. Considering the reported anabolic action of GH excess on bone with an increase in cortical thickness during the active phase and loss after treatment of the GH excess one might consider treating patients with anti-osteoporotic therapy in a quite early stage, i.e., immediately after surgery or after the first initiation of octreotide to prevent cortical bone loss. In addition, hypogonadism needs to be adequately replaced, as well as general measures to prevent bone loss, like prescription of vitamin D and calcium supplements, should be initiated.

\section{Compliance with Ethical Standards}

Conflict of Interest The authors declare that they have no competing interest.

Ethical approval This article does not contain any studies with human participants or animals performed by any of the authors.

\section{References}

1. K.M. Claessen, G. Mazziotti, N.R. Biermasz, A. Giustina, Bone and joint disorders in acromegaly. Neuroendocrinology 103(1), 86-95 (2016). (review)

2. T. Ueland, E.N. Ebbesen, J.S. Thomsen, L. Mosekilde, K. Brixen, A. Flyvbjerg, J. Bollerslev, Decreased trabecular bone biomechanical competence, apparent density, IGF-II and IGFBP-5 content in acromegaly. Eur. J. Clin. Invest. 32, 122-128 (2002)

3. M. Madeira, L.V. Neto, F. de Paula Paranhos Neto, I.C. Barbosa Lima, L.M. Carvalho de Mendonça, M.R. Gadelha, M.L. Fleiuss de Farias, Acromegaly has a negative influence on trabecular bone, but not on cortical bone, as assessed by high-resolution peripheral quantitative computed tomography. J. Clin. Endocrinol. Metab. 98, 1734-1741 (2013)

4. F. Maffezzoni, M. Maddalo, S. Frara, M. Mezzone, I. Zorza, F. Baruffaldi, F. Doglietto, G. Mazziotti, G. Maroldi, A. Giustina, High-resolution-cone beam tomography analysis of bone microarchitecture in patients with acromegaly and radiological vertebral fractures. Endocrine 54:532-542 (2016). doi:10.1007/s12020-0161078-3

5. E. Valassi, I. Crespo, J. Malouf, J. Llauger, A. Aulinas, A.M. Marín, B. Biagetti, S.M. Webb, Reduction of trabecular and cortical volumetric bone mineral density at the proximal femur in patients with acromegaly. Eur. J. Endocrinol. 174(2), 107-114 (2016). 